\title{
Identification of Emerging Brominated Chemicals as the Transformation Products of Tetrabromobisphenol A (TBBPA) Derivatives in Soil
}

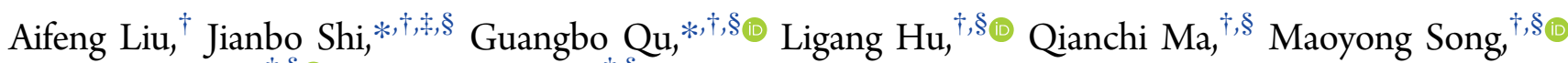 \\ Chuanyong Jing, ${ }^{\dagger, \S}$ and Guibin Jiang ${ }^{\dagger, \S}$
}

${ }^{\dagger}$ State Key Laboratory of Environmental Chemistry and Ecotoxicology, Research Center for Eco-Environmental Sciences,

Chinese Academy of Sciences, P.O. Box 2871, Beijing 100085, China

${ }^{\ddagger}$ Institute of Environment and Health, Jianghan University, Wuhan 430056, China

${ }^{\S}$ College of Resources and Environment, University of Chinese Academy of Sciences, Beijing 100049, China

Supporting Information

ABSTRACT: In contrast to the extensive investigation already conducted on tetrabromobisphenol A (TBBPA), the metabolism of TBBPA derivatives is still largely unknown. In this paper, we characterized unknown brominated compounds detected in 84 soil samples collected from sites around three brominated flame retardant production plants to determine possible transformation products of TBBPA derivatives. In addition to tribromobisphenol A (TriBBPA), dibromobisphenol A (DBBPA), and TBBPA, six novel transformation products, TriBBPA mono(allyl ether) (TriBBPA-MAE), DBBPA-MAE, hydroxyl TriBBPA-MAE, TBBPA mono(2-bromo-3-hydroxypropyl ether) (TBBPA-MBHPE), TBBPA mono(2,3-dihydroxypropyl ether) (TBBPA-MDHPE), and TBBPA mono(3-hydroxypropyl ether)

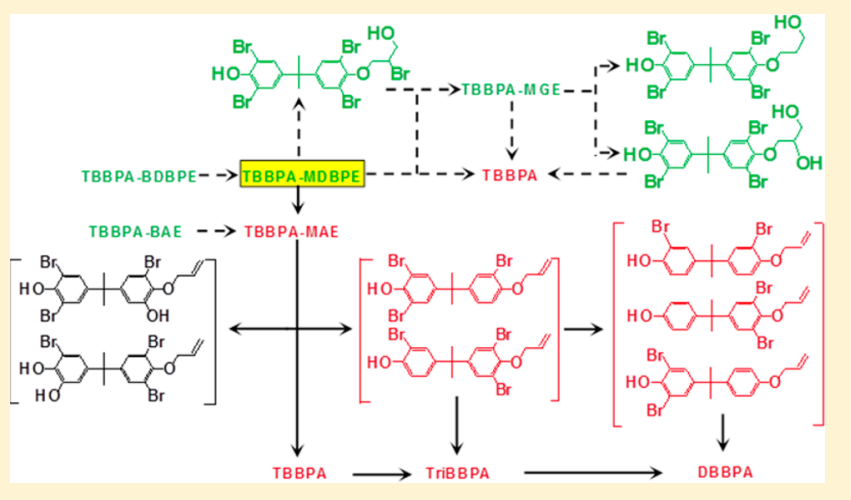
(TBBPA-MHPE) were identified. The detection frequencies of these identified chemicals in soil samples ranged from $17 \%$ to $89 \%$, indicating the widespread presence of the transformation products. To uncover the possible TBBPA derivative transformation pathways involved, super-reduced vitamin B12 (cyanocobalamin, (CCAs)) was used to treat TBBPA derivative and transformation products in this process were characterized. To our knowledge, this is the first study examining the transformation of TBBPA derivatives and the first to report several novel associated TBBPA and bisphenol A derivatives as transformation products. Our research suggests that ether bond breakage and debromination contribute to the transformation of TBBPA derivatives and the existence of the novel transformation products. These data provide new insights into the fate of TBBPA derivatives in environmental compartments.

\section{INTRODUCTION}

Tetrabromobisphenol A (TBBPA) and its derivatives are now the most widely used brominated flame retardants (BFRs) in the world, the estimates of the production volumes are rising. ${ }^{1}$ Evidence suggests that these chemicals exhibit potential nerve toxicity, reproductive toxicity, and endocrine disruption effects on bio-organisms. ${ }^{2-4}$ A wide spectrum of TBBPA derivatives have been detected in different environmental compartments. ${ }^{5-7}$ TBBPA bis(allyl ether) (TBBPA$\mathrm{BAE}$ ), TBBPA bis(2,3-dibromopropyl ether) (TBBPABDBPE), and tetrabromobisphenol $S$ bis(2,3-dibromopropyl ether) (TBBPS-BDBPE) have been identified in herring gull eggs, ${ }^{8}$ while TBBPA-BAE has been identified as the main neurotoxic agent in contaminated sites, ${ }^{2}$ and TBBPA-BDBPE has been found in atmospheric samples collected around the Great Lakes. ${ }^{9}$ These accumulated chemicals may undergo transformation through oxidation, exposure to sunlight and biological transformation, leading to the generation of unknown brominated compounds. ${ }^{10}$ Our previous work identified TBBPA derivatives including TBBPA mono(allyl ether) (TBBPA-MAE), TBBPA mono(2-bromoallyl ether) (TBBPA-MBAE), and TBBPA mono(2,3-dibromopropyl ether) (TBBPA-MDBPE), as possible degradation products or byproducts of TBBPA-BAE and TBBPA-BDBPE. ${ }^{11,12}$ Meanwhile, the potential exists for TBBPA-MAE and TBBPA-MDBPE released into the environment to be transported to remote areas, where it may accumulate in biotic systems. ${ }^{11,13}$ TBBPA mono(glycidyl ether) (TBBPA-MGE) and TBBPA mono(2-hydroxyethyl ether) (TBBPA-MHEE) have also been found in water samples as the transformation products or byproducts of TBBPA derivatives. ${ }^{14}$ Collectively, these investigations suggest that TBBPA

Received: February 27, 2017

Revised: April 18, 2017

Accepted: April 25, 2017

Published: April 25, 2017 
derivatives could transform into other brominated compounds in environmental compartments. Some of the transformation products may display greater side effects than the main products. $^{15}$

Transformation of BFRs into metabolites can occur via a number of different pathways, including debromination, ${ }^{16,17}$ O-methylation, ${ }^{18}$ ether-bond scission, ${ }^{19}$ and alkyl chain cleavage. ${ }^{13,18,20-22}$ Halogenated contaminants, including those generated via TBBPA degradation under anoxic conditions, are important products of biotransformation. As an electron acceptor in microorganisms, TBBPA was debrominated to less brominated BPA under anoxic conditions. ${ }^{23-25}$ Many bacterial strains involved in the effective dehalogenation of halogenated compounds under anoxic conditions have been reported. ${ }^{26,27}$ The anaerobic Gram-negative bacterium Sulfurospirillum multivorans (previously known as Dehalospirillum multivorans) has demonstrated great potential for the anaerobic transformation of halogenated compounds. ${ }^{28-30}$

The transformation studies of TBBPA based on isotope labeling and high resolution mass spectrometry (HRMS) have been conducted under different mimic environment condition and characterized TBBPA degradation products without standards. ${ }^{13,16,18,20,21}$ Many metabolites were characterized by HRMS in the transformation study of the BFRs and these metabolites might present in the environment to be the emerging contaminants. ${ }^{13,18,21}$ Many organic and inorganic mediates, including TBBPA sulfate, debrominated products, oxygen-methylation products, bromopenols, nitro-brominated bisphenol $\mathrm{A}$, etc. have been identified under different conditions. ${ }^{13,16,18,20,21,31,32}$ However, the environment behavior and fate of the identified chemicals have not been well studied mainly because of the lacking of commercial available standards at present. ${ }^{33}$ Meanwhile, the silico prediction is another quick, easy, and feasible way for organic pollutants transformation study and gives possible degradation products and metabolism pathway. $^{34}$

On the basis of in silico prediction, TBBPA derivatives have been shown to transform into lower brominated BFRs; TBBPA was reported as one of the most important degradation products. ${ }^{2}$ However, to our knowledge, detailed investigations of TBBPA transformation published in recent years, ${ }^{11,12,14-16,20,23,25,35,36}$ have provided no data on the transformation of TBBPA derivatives. The transformation of TBBPA derivatives therefore warrants detailed investigation into the fate of these contaminants to increase our understanding of the transformed unknowns arising from TBBPA derivatives. In this study, we demonstrate the transformation of TBBPA derivatives by characterizing unknowns found in real soil samples collected from a contaminated area. Transformation of TBBPA derivatives under super-reduced conditions was investigated to uncover the possible mechanisms involved.

\section{MATERIALS AND METHODS}

2.1. Chemicals. TBBPA (Alfa Aesar, 98\%), TBBPA-BAE (Aldrich, 99\%), and TBBPA-BDBPE (Aldrich, 99\%) were used without further purification. TBBPA-MAE, TBBPA-MBAE, TBBPA-MDBPE, TBBPA-MGE, and TBBPA-MHEE were synthesized in our previous work. ${ }^{11,12,14}$ Sodium citrate and titanium(III) chloride $(20 \% \mathrm{w} / \mathrm{v}$, in $2 \mathrm{~N} \mathrm{HCl})$ were purchased from Acros Organics. $\mathrm{Na}_{2} \mathrm{CO}_{3}$ and anhydrous $\mathrm{Na}_{2} \mathrm{SO}_{4}$ were obtained from Sinopharm Chemical Reagent Co., Ltd. Vitamin B12 (98\%) was purchased from Sigma-Aldrich. $\mathrm{D}_{10}$-labled and ring ${ }^{13} \mathrm{C}_{12}$-labled TBBPA (99\%, Cambridge Isotope Laboratories, Inc.) were used as the surrogate and internal standards (IS). The abbreviations of all the chemicals in this study were shown in Table S1 of the Supporting Information (SI). Acetone, methanol, methane dichloride (DCM), and hexane were all HPLC grade. Water was generated using a Milli-Q advantage A10 system.

2.2. Sample Collection. Soil samples were collected around three BFRs factories in Shouguang, Shandong Province; all sampling sites are shown in Figure S1. The total production of TBBPA and its derivatives in the three BFRs factories has exceeded 70000 tons/year, and one of these three plants is currently the largest BFRs supplier in China. The samples were collected in the industrial park located near the three BFRs factories and some residential villages. Relatively high concentrations of TBBPA in soil samples were found at a depth of $0-40 \mathrm{~cm} ;{ }^{37}$ therefore, we collected soil samples at each sampling site at the surface $(0-5 \mathrm{~cm}$ depth) and the beneath it $(30-40 \mathrm{~cm}$ depth). A total of 84 soil samples (taken from 42 sampling sites) were collected at a spatial distance from 0 to $12 \mathrm{~km}$ around the BFRs factories with a stainless steel scoop and packed in aluminum foil. The soil samples were freezedried, filtered through a stainless steel grid (100 mesh) and stored at $-20{ }^{\circ} \mathrm{C}$ until they could be analyzed.

2.3. Sample Pretreatment. The soil samples ( $2.0 \mathrm{~g}$ each) were mixed with anhydrous sodium sulfate $(5 \mathrm{~g})$, spiked with $\mathrm{D}_{10}$-labeled TBBPA (10 ng) surrogate standards, and extracted with $\mathrm{DCM} /$ acetone $(2 / 1, \mathrm{v} / \mathrm{v})$ on an accelerated solvent extractor (Dionex ASE 350) at $100{ }^{\circ} \mathrm{C}$ and 1500 psi. Detailed procedures are described in previous studies. ${ }^{12}$ Briefly, the extracts were purified using gel permeation chromatography (GPC), Supelclean LC-Si cartridge (500 mg, $6 \mathrm{~mL}$ ), and an ENVI-Carb cartridge (500 mg, $6 \mathrm{~mL})$. TBBPA-BAE and TBBPA-BDBPE were eluted from LC-Si cartridge, the solvent was changed to $50 \mu \mathrm{L}$ methanol, and the sample was analyzed with high performance liquid chromatography-diode array detector (HPLC-DAD). TBBPA and polar analogs were eluted using ENVI-Carb cartridge and the solvent was changed to $200 \mu \mathrm{L}$ methanol; the sample was then spiked with ${ }^{13} \mathrm{C}_{12}$-labeled TBBPA (10 ng) and analyzed with Ultrahigh performance liquid chromatography-Orbitrap high resolution mass spectrometry (UHPLC-Orbitrap HRMS).

2.4. Transformation Experiment. Preparation of CCAs: ${ }^{38-40}$ in an anaerobic glovebox, sodium citrate solution ( $50 \mathrm{~mL}, 0.4 \mathrm{M}, \mathrm{pH}=7.0$ ) was added to $15 \mathrm{~mL}$ titanium(III) chloride $(20 \% \mathrm{w} / \mathrm{v}$, in $2 \mathrm{~N} \mathrm{HCl}) ; \mathrm{Na}_{2} \mathrm{CO}_{3}$ solution $(2 \mathrm{M})$ was then added to adjust the $\mathrm{pH}$ of the solution to 8.0. Finally, the solution was diluted to $100 \mathrm{~mL}$ with deionized water. CCAs $(80 \mu \mathrm{M})$ was prepared with titanium(III) citrate. CCAs $(5 \mathrm{~mL})$ was used to expose TBBPA-MDBPE $(20 \mu \mathrm{g})$ at different durations (10 min, $20 \mathrm{~min}, 30 \mathrm{~min}, 60 \mathrm{~min}, 2$ and $24 \mathrm{~h}$ ). After transformation, samples were treated with DCM $(5 \mathrm{~mL})$ three times to extract the target compounds. The combined organic phase was eliminated with mild nitrogen gas. The residue was redissolved in $1 \mathrm{~mL}$ methanol and analyzed.

2.5. Instrumental Analysis. Ultrahigh resolution hybrid Qh-Fourier transform ion cyclotron resonance mass spectrometry (FTICR-MS, Solarix, 15.0 T, Bruker Daltonics) was used in the initial screening of transformation products. UHPLC (Ultimate 3000 UHPLC) coupled with Orbitrap Fusion Tribrid mass spectrometry (UHPLC-Orbitrap HRMS, Thermo Fisher Scientific, U.S.A.) was used for the qualitative and quantitative analysis. ZORBAX ODS $\left(150 \times 3.0 \mathrm{~mm}^{2}, 5 \mu \mathrm{m}\right.$, Agilent $)$ was 
selected for the separation of target compounds. HPLC-DAD was used for the determination of TBBPA-BAE and TBBPABDBPE in the collected samples. The instrument conditions and the optimized parameters of FTICR-MS (Table S2), UHPLC-Orbitrap HRMS and HPLC-DAD are described in detail in the SI.

2.6. Quality Control and Quality Assurance. The method performance data after optimization is shown in Table S3. The recoveries of TBBPA, TBBPA-BAE, TBBPABDBPE, TBBPA-MAE, TBBPA-MBAE, TBBPA-MDBPE, TBBPA-MGE, and TBBPA-MHEE from soil samples ranged from $78 \%$ to $95 \%$. The method detection limits (MDLs) ranged from 0.02 to $3 \mathrm{ng} / \mathrm{g}$ dry weight $(\mathrm{dw})$ for the soil samples.

Novel TBBPA analogs were identified with Orbitrap HRMS at a resolution of 120000 and mass error of $<5$ ppm. FTICRMS was used for the identification of transformation products at a resolution of 240000 , which guaranteed that the molecular formula could be determined within $1 \mathrm{ppm}$ error deviation. The molecular formula was calculated using the SmartFormula tool included in the DataAnalysis software application (Bruker Daltonics) based on the appropriate $\mathrm{m} / z$ values. Meanwhile, $\mathrm{mSigma}$ value $<100$ was another important factor in the identification of transformation products, indicating that the isotopic pattern was in accordance with the theoretical molecular formula. ${ }^{41}$

All transformation experiments were performed in triplicate. The standard deviations of the amounts of the transformation products from each sample were all less than 20\%. Blank samples and TBBPA-MDBPE-spiked controls without CCAs were prepared at the same time as the experiments. No transformation products were detected in the control samples.

2.7. Statistical Analysis. Using SPSS 11.0 software (SPSS Inc., Chicago, IL), the Spearman's rank correlation test was conducted between TBBPA analog concentrations, total organic content (TOC) and $\mathrm{pH}$ values for the soil samples. When the $p$ value is below 0.05 , the linear regression is regarded as significant between two tested variables. Concentrations below the detection limits were treated as half of the detection limit. The correlation analysis was only conducted for the chemicals with detection frequencies greater than $75 \%$.

\section{RESULTS AND DISCUSSION}

3.1. Target Analysis in Soil Samples. To determine the distribution of TBBPA derivatives, soil samples collected around the manufacturing plant were analyzed using Orbitrap-HRMS. TBBPA derivatives, including TBBPA, TBBPABAE, and TBBPA-BDBPE, and their possible transformation products or byproducts, TBBPA-MAE, TBBPA-MBAE, TBBPA-MDBPE, TBBPA-MGE, and TBBPA-MHEE (mass chromatography in Figure S2), were measured in the soil samples (for detailed data, see Table S4). The detectable concentrations of TBBPA, TBBPA-BAE and TBBPA-BDBPE were much higher than those of the other chemicals. The concentration ranges (detection frequencies) of TBBPA, TBBPA-BAE, and TBBPA-BDBPE were lower than the method detection limit (<MDL)-4.1E5 ng/g dw (99\%), 1.08-6.2E4 ng/g $\mathrm{dw}(100 \%)$ and <MDL-1.3E7 $\mathrm{ng} / \mathrm{g} \mathrm{dw}$ (95\%), respectively. These results indicated that TBBPA, TBBPA-BAE, and TBBPA-BDBPE were the BFRs produced commercially in the factories. TBBPA-MAE, TBBPA-MBAE, TBBPA-MDBPE, TBBPA-MHEE, and TBBPA-MGE were also positively detected in the soil samples with the following concentration ranges and detection frequencies for each compound: TBBPA-MAE,
$<$ MDL-1344.6 ng/g dw (94\%), TBBPA-MBAE, <MDL-21.3 ng/g dw (99\%), TBBPA-MDBPE, <MDL-1233.6 ng/g dw (94\%), TBBPA-MHEE, <MDL-13.7 ng/g dw (14\%) and TBBPA-MGE, <MDL-64.4 ng/g dw (37\%), respectively. Significant correlations $(p<0.05)$ observed between any two of the six chemicals (Table S5) indicated the same release resource for these chemicals. The concentrations of TBBPA, TBBPA-BAE, TBBPA-BDBPE, TBBPA-MAE, and TBBPAMDBPE were higher in the soil samples collected near the BRFs factories (Samples S21-S26) compared with those collected farther away (Figure S3).

The concentrations of TBBPA, TBBPA-BAE, TBBPABDBPE, TBBPA-MAE, and TBBPA-MDBPE detected in the top layer soil samples were higher than those in the sublayer samples (Figure S3). At one sampling site, the ratios of TBBPA-MAE/TBBPA-BAE or TBBPA-MDBPE/TBBPABDBPE in each layer were calculated (Figure S3). In the previous study, the ratios of TBBPA-MAE/TBBPA-BAE and TBBPA-MDBPE/TBBPA-BDBPE in the commercial products of the BFRs factories were $9.2 \times 10^{-03}: 1$ and $5.4 \times 10^{-03}: 1$, respectively. In the top layer samples, ratios of TBBPA-MAE/ TBBPA-BAE and TBBPA-MDBPE/TBBPA-BDBPE were similar to those in commercial products, while the ratios in the sublayer were higher than this value. These results suggested that certain portions of TBBPA-MAE or TBBPA-MDBPE were transformed from TBBPA-BAE or TBBPA-BDBPE under the anoxic condition.

3.2. Identification of Unknowns in Real Soil Samples. To determine whether transformation of TBBPA derivatives was occurring in the environment, soil samples collected around the manufacturing plant were analyzed using OrbitrapHRMS. Orbitrap-HRMS provides suitable sensitivity and accuracy for the identification of TBBPA derivatives in the environment ${ }^{14,15}$ and is a powerful tool for the characterization of unknown chemicals. In addition, the $\mathrm{m} / z$ values of the analytes obtained are more accurate, and the mass sensitivity is higher than traditional mass spectrometry. ${ }^{14,42}$ On the basis of the structures of the most important TBBPA derivatives, TBBPA byproducts or their possible transformation products, ${ }^{36}$ we proposed the following rules for the characterization of unknown TBBPA analogs in the soil samples: (1) When the number of $\mathrm{C}$ atoms was between 15 and 18 and the number of $\mathrm{H}$ atoms was greater than 10 , the chemicals might be brominated bisphenol analogs; and (2) when the number of $\mathrm{C}$ atoms was 6, the total number of $\mathrm{H}$ and $\mathrm{Br}$ atoms was 6 , the chemicals were considered to be bromophenol candidates.

As shown in Table 1, 14 nontargeted brominated compounds (NOs.1-14) were detected in the soil samples according to full scan mass spectrometry, of which 8 chemicals were similar to TBBPA (Figure 1). Chemical NO.1 had an isotope ratio of $1: 5: 10: 10: 5: 1$, indicating $5 \mathrm{Br}$ atoms were present in its structure, and its molecular formula was $\left[\mathrm{C}_{18} \mathrm{H}_{16} \mathrm{O}_{3} \mathrm{Br}_{5}\right]^{-}$. Chemical NOs.2, 3, and 4 had an isotope ratio of $1: 4: 6: 4: 1$, indicating that four $\mathrm{Br}$ atoms were present in the structures of these compounds; the molecular formulas calculated from the detected $m / z$ values were $\left[\mathrm{C}_{18} \mathrm{H}_{17} \mathrm{O}_{4} \mathrm{Br}_{4}\right]^{-},\left[\mathrm{C}_{18} \mathrm{H}_{17} \mathrm{O}_{3} \mathrm{Br}_{4}\right]^{-}$, and $\left[\mathrm{C}_{16} \mathrm{H}_{13} \mathrm{O}_{2} \mathrm{Br}_{4}\right]^{-}$, respectively. As shown in Figure 2, the $\mathrm{MS}^{2}$ spectra of chemicals NO.1-4 were the same as TBBPA-MAE and TBBPA-MDBPE (Figure S4), indicating that these four chemicals possessed structural skeletons similar to TBBPA-MAE or TBBPA-MDBPE. On the basis of these observations, chemicals NOs.1-3 were identified as monomodified TBBPA derivatives, TBBPA mono(2-bromo-3-hydroxypropyl ether) 
H
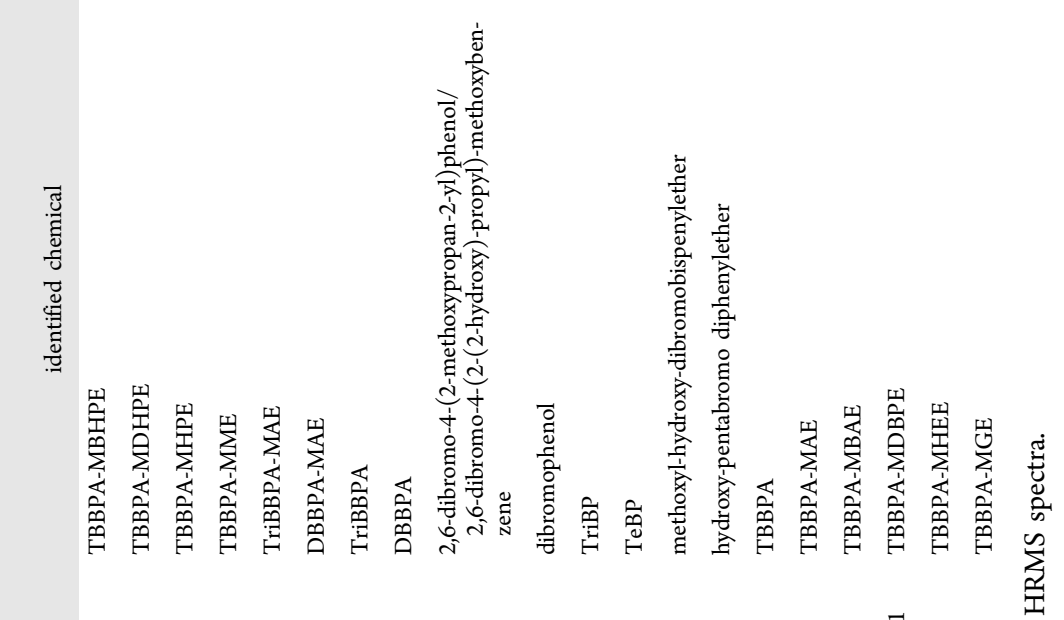

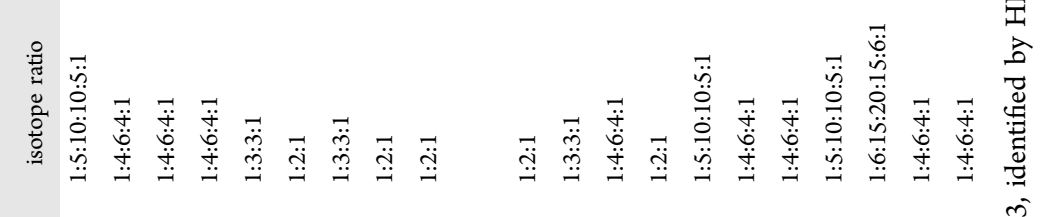

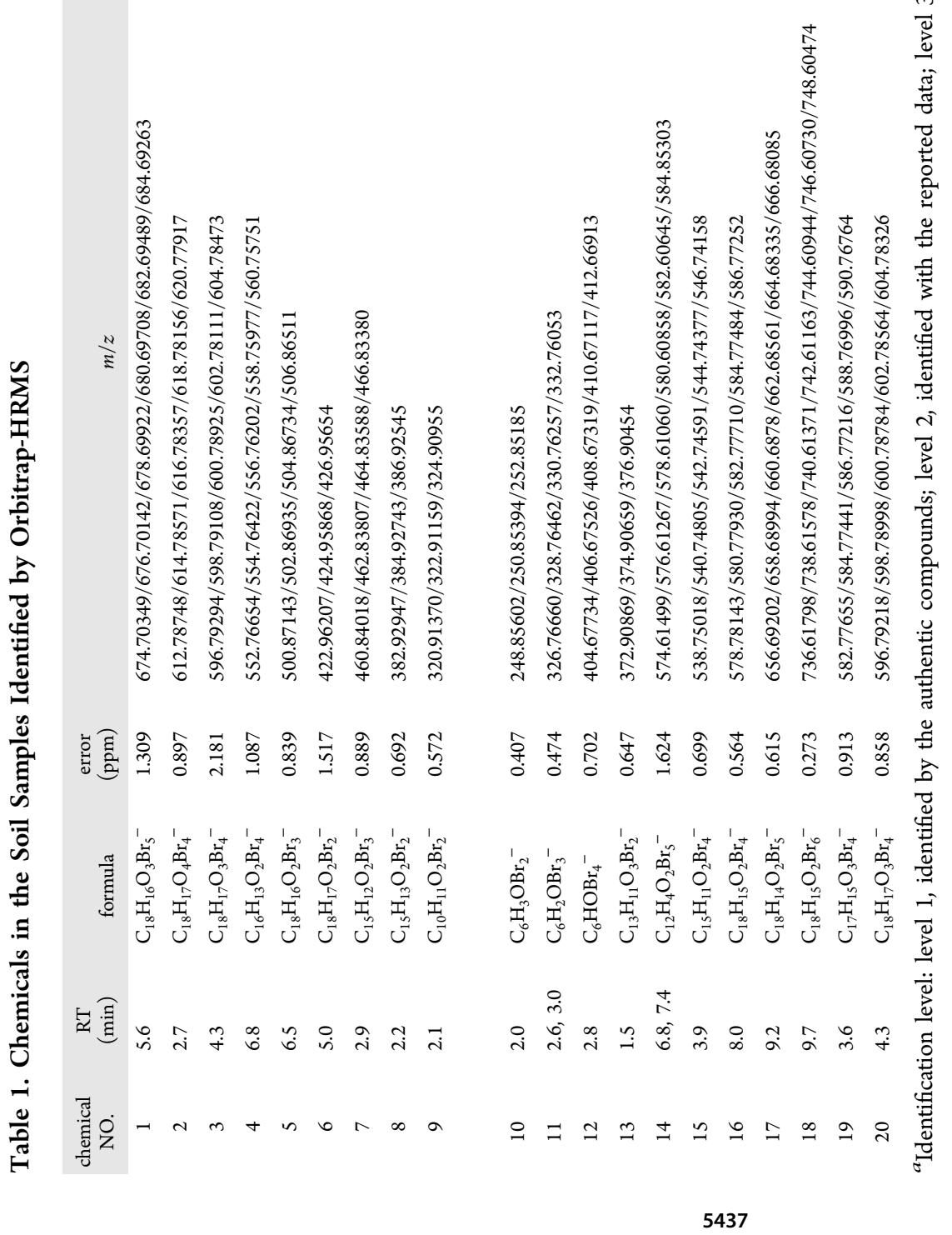




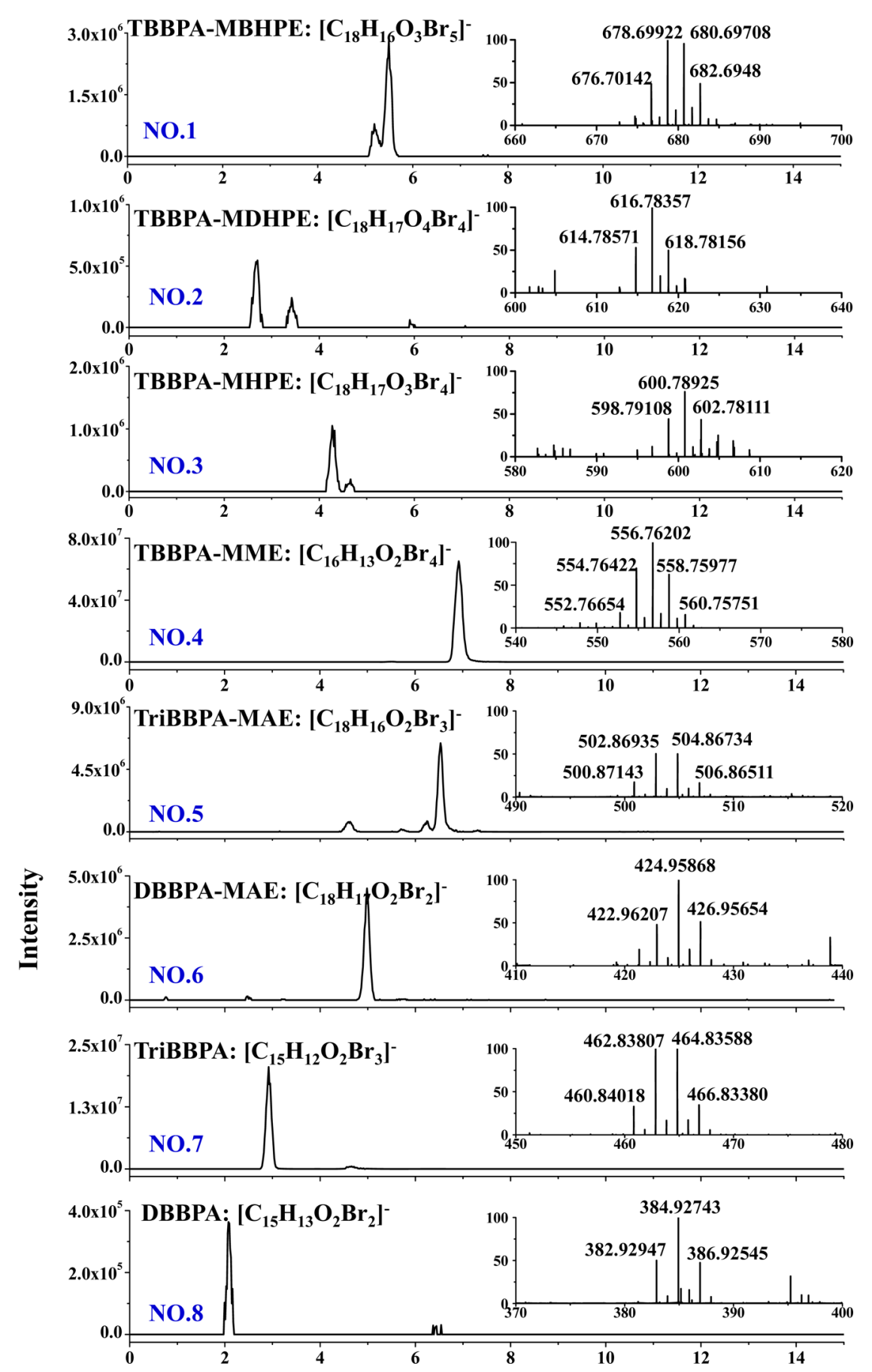

Figure 1. Mass chromatography and spectra of TBBPA analogs in soil samples. TBBPA-MDHPE, TBBPA-MBHPE, TBBPA-MME, and TriBBPA-MAE in sample S24-1; DBBPA-MAE in sample S5-2; TBBPA-MHPE, TriBBPA, and DBBPA in sample S26-1.

(TBBPA-MBHPE), TBBPA mono(2,3-dihydroxypropyl ether) (TBBPA-MDHPE), and TBBPA mono(3-hydroxypropyl ether) (TBBPA-MHPE) and this finding was further confirmed with synthesized standards (as outlined in the next section). Chemical NO.4 may have been TBBPA mono(methyl ether) (TBBPA-MME), which has been detected previously in TBBPA transformation processes (for detailed data, see the mass spectra in Figures 1 and 2). ${ }^{18}$

Some lower brominated BPA derivatives were also detected in the soil samples (chemicals NO.5-8 in Table 1 and Figure 1). The isotope ratios of chemicals NO.5 and NO.7 were 1:3:3:1, indicating the presence of three $\mathrm{Br}$ atoms in their structures.
Their calculated molecular formulas were $\left[\mathrm{C}_{18} \mathrm{H}_{16} \mathrm{O}_{2} \mathrm{Br}_{3}\right]^{-}$and $\left[\mathrm{C}_{15} \mathrm{H}_{12} \mathrm{O}_{2} \mathrm{Br}_{3}\right]^{-}$, respectively. For chemicals NO.6 and NO.8, the isotope ratios were 1:2:1, suggesting two $\mathrm{Br}$ atoms in their structures. Their calculated molecular formulas were $\left[\mathrm{C}_{18} \mathrm{H}_{17} \mathrm{O}_{2} \mathrm{Br}_{2}\right]^{-}$and $\left[\mathrm{C}_{15} \mathrm{H}_{13} \mathrm{O}_{2} \mathrm{Br}_{2}\right]^{-}$, respectively. As shown in Figure 2, fragmentation of chemical NO.5 and NO.6, due to the loss of $\mathrm{C}_{3} \mathrm{H}_{5}$ and $\mathrm{CH}_{3}$, formed ion clusters at $m / z 446.8 / 448.8$ and 368.9. This was similar to the formation of ion clusters observed at $m / z 526.7$ due to the fragmentation of TBBPA-MAE (Figure S4). Therefore, chemicals NO.5 and NO.6 were identified as tribromobisphenol A mono(allyl ether) (TriBBPA-MAE) and DBBPA-MAE, respectively. Analogously, chemicals NO.7 


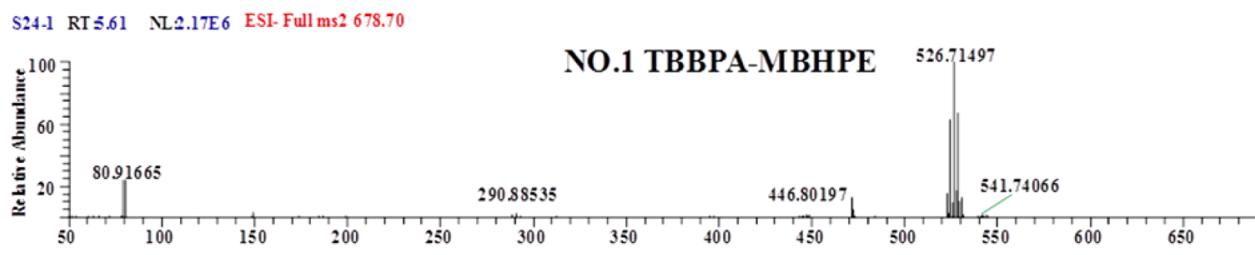
S24-1 RT:2.73 NL S.47E5 ESI- Full ms2 616.78

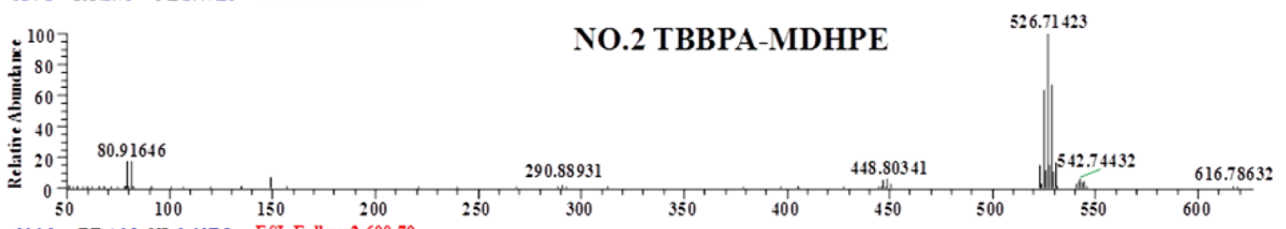
S26-1 RT:4.28 NL.3.63E5 ESI-Full ms2 600.79

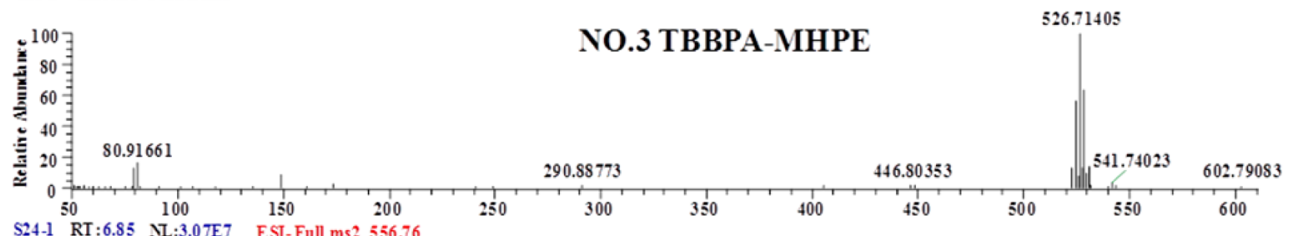
S24-1 RT:6.85 $\quad$ TL:3.07E7 EST-Full ms2 556.76

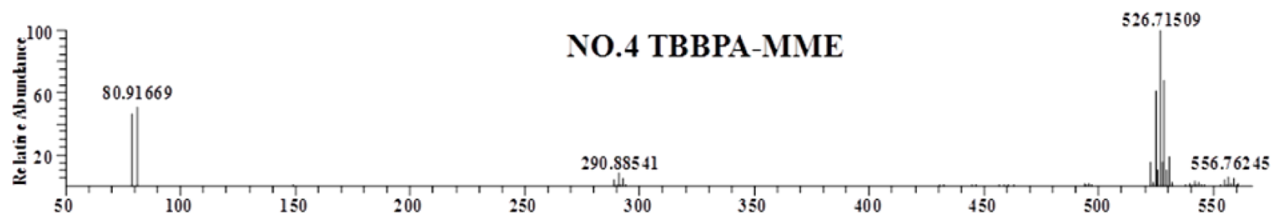
S24-1 RT:6.51 NL3.69E6 ESI-Full ms2 502.87

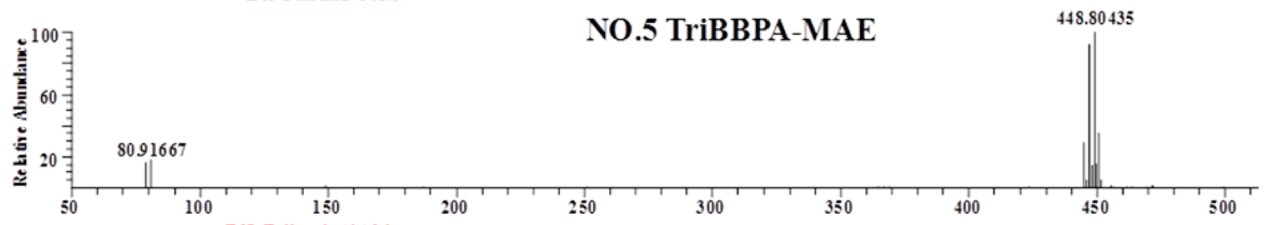
S5-2 RT:5.24 NL2 \$3E6 ESI-Full ms2 42496

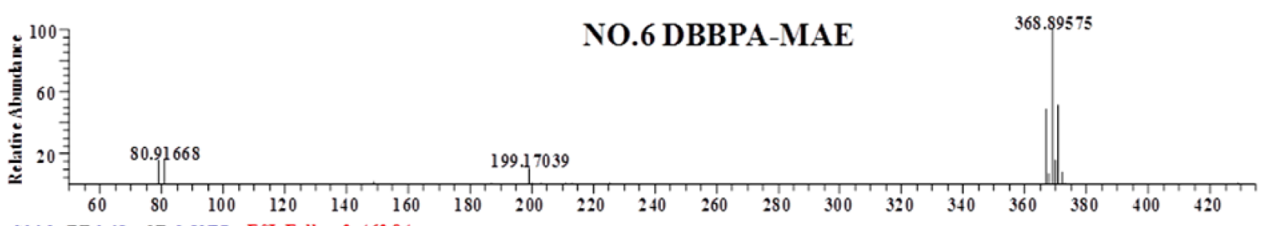
S26-1 RT:2.65 NI:1.53E7 ESI-Full ms2 462.84

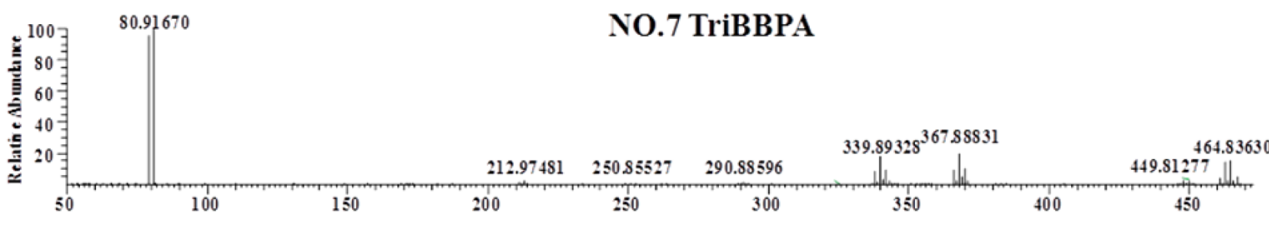
$\begin{array}{crr}\text { S26-1 RT2.09 NL2.16E6 } & \text { ESI-Full ms2 } 38493 & \text { NO.8 DBBPA } \\ 80.91668 & 165.97911 & \end{array}$

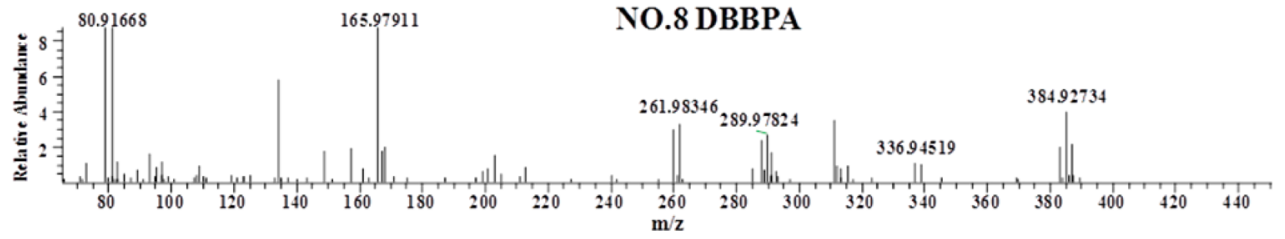

Figure 2. $\mathrm{MS}^{2}$ spectra of nontargeted TBBPA analogs in soil samples.

and NO.8 formed fragment ion clusters (Figure 2) similar to those of TBBPA (Figure S5). The Br atoms of TBBPA were gradually substituted with hydrogen atoms to form chemicals NO.7 and NO.8, and they were identified as TriBBPA and DBBPA, which have previously been identified as metabolites in TBBPA transformation studies. ${ }^{20,43-45}$ The detection frequencies of TBBPA-MME, TriBBPA-MAE, DBBPA-MAE, TriBBPA, and DBBPA were 99\%, 31\%, 23\%, 89\%, and 49\%, respectively. Detailed information for the nontargeted TBBPA analogs is shown in Table S6. It has been reported that the main degradation pathway of TBBPA in the environment is debromination of the aryl ring which forms less brominated BPA, including TriBBPA, DBBPA, monobromobisphenol A (MBBPA), and BPA, ${ }^{13,16,18,20}$ and the concentration and detection frequency of TriBBPA in human breast milk were higher than TBBPA, DBBPA, and MBBPA. ${ }^{46,47}$ In this study, TBBPA-MME and TriBBPA showed higher detection frequencies and higher MS signal ratios than the other chemicals, but the signal 
intensities observed were much lower than that of TBBPA. The detection frequencies for TriBBPA and DBBPA were higher than TBBPA-MAE and DBBPA-MAE, respectively. All of these results indicated that TBBPA-MME and TriBBPA were the main environmental degradation products and derivatives of TBBPA.

Chemical NO.9 in Table 1 contains two $\mathrm{Br}$ atoms and its molecular formula was $\left[\mathrm{C}_{10} \mathrm{H}_{11} \mathrm{O}_{2} \mathrm{Br}_{2}\right]^{-}$. The $\mathrm{MS}^{2}$ spectrum (Figure $\mathrm{S} 6$ ) was the same as the laccase-catalyzed TBBPA transformation product 2,6-dibromo-4-(2-methoxypropan-2yl)phenol. ${ }^{48}$ Meanwhile, we could not exclude the methylation of phenol and the formation of 2,6-dibromo-4-(2-(2-hydroxy)propyl)-methoxybenzene. ${ }^{15,18,21}$ This substance may be related to the degradation of TBBPA in the soil. The isotope ratios and calculated molecular formulas of Chemical NOs.10-12 were in accordance with dibromophenol (DBP), tribromophenol (TBP), and tetrabromophenol (TeBP). Since the sampling sites were located inside the BFRs industrial park, these three bromophenols could be raw materials, byproducts, or transformation products from the production of BFRs. ${ }^{15,41,49}$ Meanwhile, polybrominated diphenyl ethers (PBDEs) analogs were detected in the nontarget scan of the soil samples. The isotope ratios of chemicals NO.13 and NO.14 indicated that the number of $\mathrm{Br}$ atom in their structures were 2 and 5, respectively; meanwhile, the calculated molecular formulas were $\left[\mathrm{C}_{13} \mathrm{H}_{11} \mathrm{O}_{3} \mathrm{Br}_{2}\right]^{-}$and $\left[\mathrm{C}_{12} \mathrm{H}_{4} \mathrm{O}_{2} \mathrm{Br}_{5}\right]^{-}$, respectively. These chemicals may be two kinds of PBDEs analogs, methoxylhydroxy-dibromodipenylether (MHDBDE) and hydroxy-pentabromodiphenylether (HPBDE). The distribution results for these bromophenols and PBDEs analogs could not be obtained without standards, but they could be qualitatively determined by the top two high $m / z$ values. Their detection frequencies were calculated to be 94\% (DBP), 99\% (TBP), 7\% (TeBP), $50 \%$ (MHDBDE) and 54\% (HPBDE) by the comparison of detectable sample number to all the sample number (84).

3.3. Transformation of TBBPA-MDBPE under CCAs Conditions. The anaerobic Gram-negative bacterium, Sulfurospirillum multivorans could positively degrade halogenated compounds. After the corrinoid cofactor (norpseudo-B12) of this bacterium was identified, ${ }^{28,30}$ commercially available corrionid compound, cyanocobalamin (vitamin B12, CCA) in super-reduced state (CCAs), was used as its substitute to study the transformation mechanism of halogenated contaminants. Using CCAs, the transformations of toxaphene, ${ }^{38}$ brominated diphenyl ether, ${ }^{39}$ chlorinated ethene, ${ }^{50}$ 2,3-dibromopropyl2,4,6-tribromophenyl ether (DPTE) and other chemicals have been investigated. ${ }^{40}$

To study the transformation mechanism of TBBPA derivatives and detect the presence of novel TBBPA derivatives, CCAs in water was applied to treat TBBPA derivatives for anaerobic transformation. Regarding the investigation on TBBPA derivatives transformation, the selection of initial transformation parent chemicals was important. Since the analysis method for TBBPA-BDBPE and some possible metabolites of bis-modified TBBPA derivatives are unavailable due to the low ionization efficiency, ${ }^{35,51}$ TBBPA-BDBPE was not suitable for the initial chemical to be treated by CCAs. Therefore, TBBPA-MDBPE was applied as the representative initial chemicals to investigate the transformation of TBBPA derivatives. Thus, the transformation study of TBBPA-MDBPE could reflect the results of TBBPA-BDBPE appropriately and help identified more transformation products.
After transformation, FTICR-MS was applied for characterization under full scan mode (Figure S7), and the identified chemicals were defined as compounds $\mathrm{A}-\mathrm{H}$ (Figure S7A-H, A was TBBPA-MDBPE). The detection of ion clusters of compound $\mathrm{B}$ at $m / z 578.8 / 580.8 / 582.8 / 584.8 / 586.8$ with an isotope ratio of $1: 4: 6: 4: 1$ suggested four bromine atoms in the molecular formula (Figure S7B) and the molecular formula was found to be $\left[\mathrm{C}_{18} \mathrm{H}_{15} \mathrm{Br}_{4} \mathrm{O}_{2}\right]^{-}$. The calculated molecular formula of the compound had the same elemental composition as TBBPA-MAE, which was further confirmed using UHPLCOrbitrap HRMS (Figure S8) and TBBPA-MAE standard (Figure S2). As with compound $\mathrm{C}$, the ion clusters at $\mathrm{m} / z$ 500.9/502.9/504.9/506.9 (isotope ratio 1:3:3:1) were calculated to be $\left[\mathrm{C}_{18} \mathrm{H}_{16} \mathrm{Br}_{3} \mathrm{O}_{2}\right]^{-}$(Figure S7C), identified as TriBBPA-MAE, which was formed due to the loss of $\mathrm{Br}$ and addition of $\mathrm{H}$ to TBBPA-MAE (compound B). Compound D (Figure S7D) generated ion clusters at $m / z$ 423.0/425.0/427.0 (isotope ratio, 1:2:1); its molecular formula was determined to be $\left[\mathrm{C}_{18} \mathrm{H}_{17} \mathrm{Br}_{2} \mathrm{O}_{2}\right]^{-}$, and the compound was identified as dibromobisphenol A mono(allyl ether) (DBBPA-MAE). Ion clusters of compound E (Figure S7E) were generated at $\mathrm{m} / z$ 516.9/ $518.9 / 520.9 / 522.9(1: 3: 3: 1)$, and its molecular formula was calculated as $\left[\mathrm{C}_{18} \mathrm{H}_{16} \mathrm{Br}_{3} \mathrm{O}_{3}\right]^{-}$. This compound displayed an additional $16 \mathrm{~m} / \mathrm{z}$ units when compared with compound $\mathrm{C}$. Because the bromine atom on the aromatic ring of BFRs was a reaction site which could be hydrolyzed and substituted with a hydroxyl, ${ }^{45,52}$ the added $16 \mathrm{~m} / z$ units were usually identified as the substitution of $-\mathrm{OH}(17)$ at the aromatic ring. ${ }^{52,53}$ Hydroxyl TriBBPA has been identified previously in a TBBPA transformation study through the hydrolysis reaction ${ }^{45}$ and in soil samples with amendment of a bacterium. ${ }^{21}$ Therefore, compound $\mathrm{E}$ was identified as hydroxyl TriBBPA-MAE (HTriBBPA-MAE) generated from TBBPA-MAE. However, hydroxylation of TriBBPA-MAE (compound C) could not be ruled out as the possible transformation pathway.

Ion clusters of compound F occurred at $\mathrm{m} / z$ 538.7/540.7/ $542.7 / 544.7 / 546.7(1: 4: 6: 4: 1)$ and the calculated molecular formula was $\left[\mathrm{C}_{15} \mathrm{H}_{11} \mathrm{Br}_{4} \mathrm{O}_{2}\right]^{-}$, suggesting the presence of TBBPA, which was confirmed with UHPLC-Orbitrap-HRMS (Figure S8F, Figure S2). The formation of TBBPA could be explained by ether bond cleavage of TBBPA-MDBPE or TBBPA-MAE. Debromination is one of the most common degradation pathways of TBBPA. ${ }^{23,43}$ Regarding anaerobic transformation of TBBPA, the gradual debromination of the aromatic ring produced less brominated bisphenol A (BPA). ${ }^{16,20}$ The same transformation feature was also observed in the transformation process of TBBPA-MDBPE. As shown in Figure S7, the molecular formulas of compounds $\mathrm{G}$ and $\mathrm{H}$ were $\left[\mathrm{C}_{15} \mathrm{H}_{12} \mathrm{Br}_{3} \mathrm{O}_{2}\right]^{-}$and $\left[\mathrm{C}_{15} \mathrm{H}_{13} \mathrm{Br}_{2} \mathrm{O}_{2}\right]^{-}$, indicating that the loss of one $\mathrm{Br}$ and the subsequent addition of one $\mathrm{H}$ had occurred. The detailed speculation regarding the structures of the degradation products is presented in the SI.

The transformation tendency of TBBPA-MDBPE was shown in Figure S9A,B based on the mass spectrum peak area of the analytes compared with the original molar amount of TBBPAMDBPE. TBBPA-MDBPE $(20 \mu \mathrm{g}, 26.9 \mathrm{nmol})$ was transformed into metabolites at a ratio of $74.1 \%$ at $10 \mathrm{~min}$ and $97.3 \%$ at $24 \mathrm{~h}$, respectively (Figure S9B). Taken together, this process degraded TBBPA-MAE/TBBPA and TriBBPA-MAE/TriBBPA to their respective debrominated counterparts. Then bromine atoms on the aromatic ring were further substituted by hydrogen to form DBBPA-MAE and DBBPA with prolonged incubation. 
HTriBBPA-MAE, as the hydroxylated compound, was detected within $2 \mathrm{~h}$.

3.4. Identification of TBBPA-MHPE, TBBPA-MDHPE, and TBBPA-MBHPE in Soil Samples. It should be noted that chemicals NO.1-3 were not found as the transformation products of TBBPA-MDBPE under super-reduced conditions. To confirm the presence of these chemicals in soil samples, we synthesized TBBPA-MHPE, TBBPA-MDHPE, and TBBPA-MBHPE in the laboratory. The synthesis procedures, HRMS data and ${ }^{1} \mathrm{HNMR}$ spectra are shown in the SI (Figures S10 and S11). Because the phenol hydroxyl is easily ionized, TBBPA-MHPE, TBBPAMDHPE, and TBBPA-MBHPE generated $[\mathrm{M}-\mathrm{H}]^{-}$ions under $\mathrm{ESI}^{-}$mode with the specific $\mathrm{Br}$ isotopic characteristic. The detected $\mathrm{m} / z$ values of synthesized TBBPA-MHPE, TBBPAMDHPE, and TBBPA-MBHPE were 600.78748, 616.78241, and 678.69812/680.69598, respectively; the mass errors between the detected and calculated values were less than $2 \mathrm{ppm}$. The retention times, isotope ratios and $\mathrm{MS}^{2}$ spectra (data not shown) of the synthesized TBBPA-MHPE, TBBPA-MDHPE, and TBBPA-MBHPE were the same as that detected in the soil samples (Figure 1). Therefore, the three synthesized chemicals could be used as standards for further identification of these substances in the soil samples.

The concentrations of TBBPA-MHPE, TBBPA-MDHPE and TBBPA-MBHPE in soil samples ranged from $<\mathrm{MDL}$ to $178.8 \mathrm{ng} / \mathrm{g} \mathrm{dw}$, from $<\mathrm{MDL}$ to $20.4 \mathrm{ng} / \mathrm{g} \mathrm{dw}$, and from $<\mathrm{MDL}$ to $85.0 \mathrm{ng} / \mathrm{g} \mathrm{dw}$, respectively. The detection frequencies were $25 \%, 17 \%$, and 50\%. TBBPA-MHPE, TBBPA-MDHPE, and TBBPA-MBHPE were not detectable in the commercial products, which was a striking finding as it indicates the source of these chemicals was transformation. According to the degradation pathways (Figure 3) of TBBPA-BDBPE and TBBPA-BAE predicted by EAWAG-BBD Pathway Prediction System (http://eawag-bbd.ethz.ch/predict/), TBBPA-MDHPE and TBBPA-MBHPE can occur in the environment due to the hydrolysis of TBBPA-MGE. TBBPA-MGE has been identified as a byproduct of brominated epoxy resins. ${ }^{25}$ The concentration of TBBPA-MGE ranged from ND to $64.4 \mathrm{ng} / \mathrm{g} \mathrm{dw}$ in soil samples. On the basis of its chemical properties, the epoxy group of TBBPA-MGE is sensitive to acidic or basic conditions, which stimulate the break of the epoxy ring to form TBBPAMHPE or TBBPA-MDHPE. Thus, TBBPA-MHPE and TBBPAMDHPE might be the hydrolysis products of TBBPA-MGE in the environment. Taken together, through the analysis of the soil samples using UHPLC-HRMS and the synthesized standards, we could further confirm the presence of TBBPA-MHPE, TBBPAMDHPE, and TBBPA-MBHPE.

\section{METABOLISM, FATE, AND ENVIRONMENTAL IMPLICATIONS OF TBBPA DERIVATIVES}

In soil samples, the ratios of TBBPA-MAE/TBBPA-BAE and TBBPA-MDBPE/TBBPA-BDBPE found in the sublayer were higher than those in the surface layer, which suggests that monomodified TBBPA derivatives may be generated under anoxic conditions. As a representative monomodified TBBPA derivative, our results indicated that TBBPA-MDBPE could be anaerobically transformed via debromination and ether bond breakage. TBBPA was found to be one of the most important transformation products of TBBPA derivatives. Reported degradation products of TBBPA, TriBBPA and DBBPA were also identified in real soil samples. More importantly, six novel TBBPA derivatives including TriBBPA-MAE, DBBPA-MAE, HTriBBPA-MAE, TBBPA-MBHPE, TBBPA-MDHPE, and TBBPA-MHPE were identified for the first time (Figure 3).

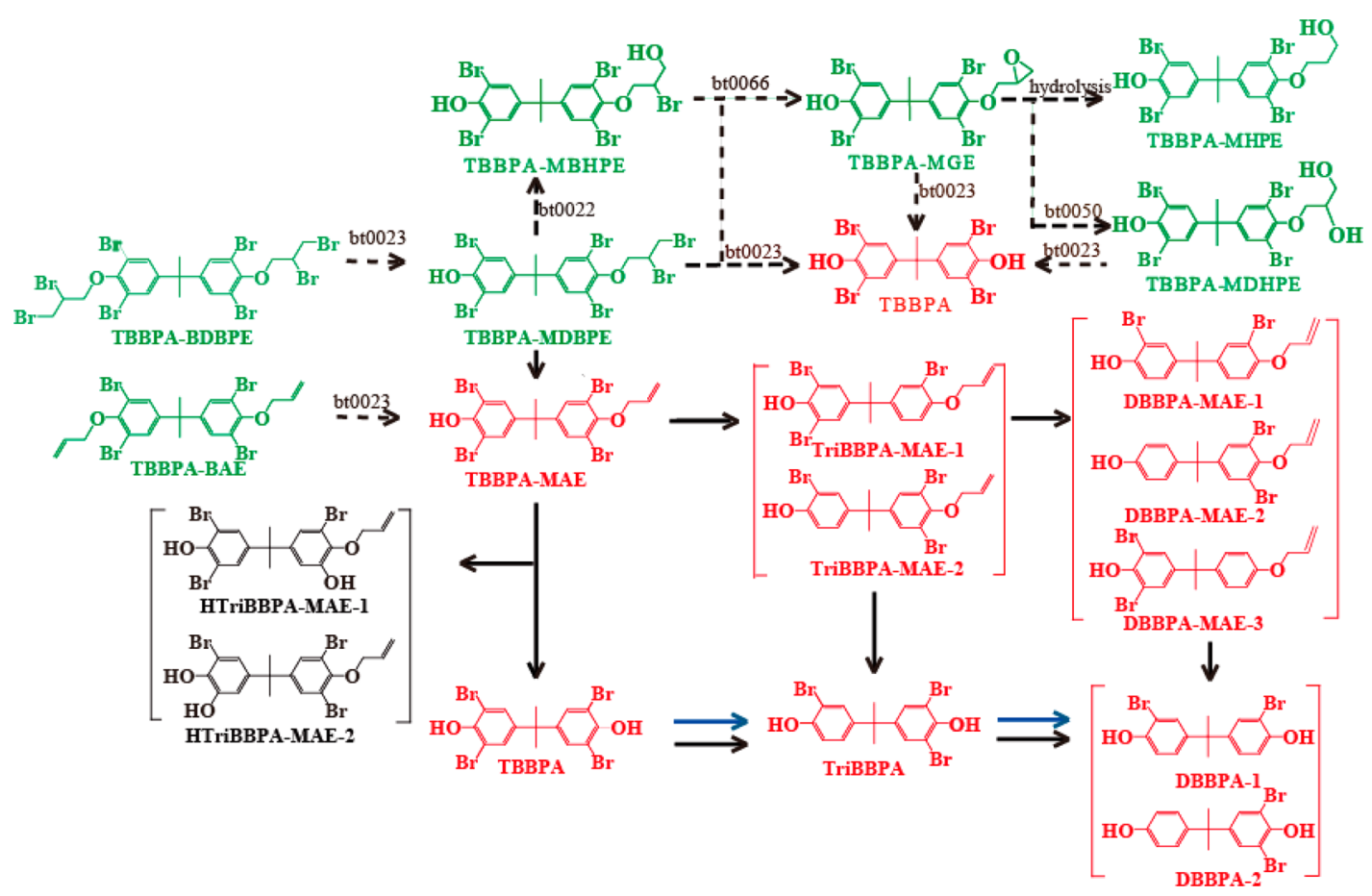

Figure 3. Anaerobic transformation pathway of TBBPA-MDBPE under CCAs conditions (solid arrow) and predicted by EAWAG-BBD Pathway Prediction System (dashed arrow). The transformation rules (bt0022-66) can be found at http://eawag-bbd.ethz.ch/predict/. Chemicals shown in red were products identified in both laboratory transformation experiments and soils samples. Chemicals shown in green were detected in the soil samples but not in transformation experiment. Chemicals shown in black were detected only in the transformation process. The pathways indicated with blue arrows were also reported in other literature. ${ }^{16,20,43}$ 
The TBBPA-MHPE, TBBPA-MDHPE, and TBBPA-MBHPE detected in the soil samples may be due to the transformation of TBBPA-MGE and TBBPA-MDBPE. TriBBPA-MAE, DBBPA-MAE, HTriBBPA-MAE, TriBBPA, and DBBPA were characterized as the transformation products of TBBPAMDBPE. TBBPA-BDBPE was detected in the soil samples with the concentration of higher than $1 \mathrm{mg} / \mathrm{g} \mathrm{dw}$, indicating its high probability of transforming to monomodified products and other complex dimodified products in the environment. According to the transformation pathways of TBBPA-MDBPE, TBBPA-BAE, and some less brominated compounds, e.g., TriBBPA-BAE, DBBPA-BAE might be the transformation products of TBBPA-BDBPE. Further transformation study concerned the fate of TBBPA-BDBPE in environment should be conducted with the development of identification methods.

As an in silico method, the US EPA EPI suite has frequently been used for calculating the physical/chemical properties of contaminants and providing the related parameters of TBBPA analogs in this study (Table S7). The ether-O linked by the 2,3-dibromopropyl group and the allyl group showed higher log $K_{\text {ow }}$ values than the other compounds. The log $K_{\text {ow }}$ values of TBBPA-BAE and TBBPA-BDBPE were higher than other TBBPA derivatives, including the transformation products. The compounds with alkyl side chains all showed log $K_{\text {ow }}$ values $>5$. Log $K_{\text {ow }}$ values decreased with decreasing $\mathrm{Br}$ numbers in the TBBPA-associated compounds. High $K_{\mathrm{oa}}$ values (close to 20) for TBBPA derivatives and monomodified chemicals indicate low respiratory elimination rates and high bioaccumulation potential in respiratory organisms. The chemicals displayed $K_{\mathrm{oc}}$ values comparable to TBBPA, indicating these compounds could be adsorbed in sediments and soils in considerable amounts through adsorption mechanisms similar to that of TBBPA. Bioconcentration factor (BCF) values decreased with the decreasing $\mathrm{Br}$ numbers at the aryl ring. For the largely produced TBBPA derivatives, TBBPA$\mathrm{BAE}$, and TBBPA-BDBPE, the BCF values were lower than $500 \mathrm{~L} / \mathrm{kg}$, wet-wt, indicating their lower bioconcentration potential. However, some TBBPA analogs with phenolic hydroxyl group, e.g., TriBBPA and TBBPA showed BCF > 5000. The BCFs of HTriBBPA-MAE and DBBPA-MAE were 10,150 and 14,910 , respectively, which was similar to or higher than the BCF value of TBBPA $(10,580)$. With respect to the potential toxicity, many of the contaminants generated by degradation exhibited more severe toxicity than the parent BFRs, including the degradation products of PBDEs and OH-PBDEs, which were more toxic than their parent compounds. ${ }^{54-56}$ The aquatic toxicity of TriBBPA, DBBPA, and MBBPA were much higher than that of TBBPA. ${ }^{57}$ Therefore, risk assessment of environmental exposure and the resulting health impacts of the transformation products of TBBPA derivatives warrant further detailed investigation.

\section{ASSOCIATED CONTENT}

\section{(S Supporting Information}

The Supporting Information is available free of charge on the ACS Publications website at DOI: 10.1021/acs.est.7b01071.

Abbreviation and structure of the chemicals; instrument parameters for FTICR-MS; the performance method data; concentration of all samples; correlation test results; map of sampling sites; mass chromatography of standards; FTICR-MS spectra, Orbitrap-HRMS chromatography and $\mathrm{MS}^{2}$ spectra of transformation products of
TBBPA-MDBPE; and transformation ratios at different time period (PDF)

\section{AUTHOR INFORMATION}

\section{Corresponding Authors}

*Tel/fax: +86-10-62849129 E-mail: jbshi@rcees.ac.cn (J.S.).

*Tel/fax: +86-10-62849129 E-mail: gbqu@rcees.ac.cn (G.Q.). ORCID

Guangbo Qu: 0000-0002-5220-7009

Ligang Hu: 0000-0002-6213-4720

Maoyong Song: 0000-0002-6776-4705

Chuanyong Jing: 0000-0001-5929-0290

Notes

The authors declare no competing financial interest.

\section{ACKNOWLEDGMENTS}

This work was supported by the National Natural Science Foundation of China (Nos. 21377155 and 21621064), the Strategic Priority Research Program of the Chinese Academy of Sciences (No. XDB14010400), and the China Postdoctoral Science Foundation (2016M602210).

\section{REFERENCES}

(1) Covaci, A.; Voorspoels, S.; Abdallah, M. A.-E.; Geens, T.; Harrad, S.; Law, R. J. Analytical and environmental aspects of the flame retardant tetrabromobisphenol-A and its derivatives. J. Chromatogr. A 2009, 1216 (3), 346-363.

(2) Qu, G. B.; Shi, J. B.; Wang, T.; Fu, J. J.; Li, Z. N.; Wang, P.; Ruan, T.; Jiang, G. B. Identification of tetrabromobisphenol a diallyl ether as an emerging neurotoxicant in environmental samples by bioassaydirected fractionation and HPLC-APCI-MS/MS. Environ. Sci. Technol. 2011, 45 (11), 5009-5016.

(3) McCormick, J. M.; Paiva, M. S.; Haeggblom, M. M.; Cooper, K. R.; White, L. A. Embryonic exposure to tetrabromobisphenol A and its metabolites, bisphenol A and tetrabromobisphenol A dimethyl ether disrupts normal zebrafish (Danio rerio) development and matrix metalloproteinase expression. Aquat. Toxicol. 2010, 100 (3), 255-262.

(4) Van der Ven, L. T.; Van de Kuil, T.; Verhoef, A.; Verwer, C. M.; Lilienthal, H.; Leonards, P. E.; Schauer, U. M.; Canton, R. F.; Litens, S.; De Jong, F. H.; Visser, T. J.; Dekant, W.; Stern, N.; Hakansson, H.; Slob, W.; Van den Berg, M.; Vos, J. G.; Piersma, A. H. Endocrine effects of tetrabromobisphenol-A (TBBPA) in Wistar rats as tested in a one-generation reproduction study and a subacute toxicity study. Toxicology 2008, 245 (1-2), 76-89.

(5) Covaci, A.; Harrad, S.; Abdallah, M. A. E.; Ali, N.; Law, R. J.; Herzke, D.; de Wit, C. A. Novel brominated flame retardants: A review of their analysis, environmental fate and behaviour. Environ. Int. 2011, 37 (2), 532-556.

(6) Cruz, R.; Cunha, S. C.; Casal, S. Brominated flame retardants and seafood safety: A review. Environ. Int. 2015, 77, 116-131.

(7) Vandermeersch, G.; Lourenco, H. M.; Alvarez-Munoz, D.; Cunha, S.; Diogene, J.; Cano-Sancho, G.; Sloth, J. J.; Kwadijk, C.; Barcelo, D.; Allegaert, W.; Bekaert, K.; Fernandes, J. O.; Marques, A.; Robbens, J. Environmental contaminants of emerging concern in seafood - European database on contaminant levels. Environ. Res. 2015, 143, 29-45.

(8) Letcher, R. J.; Chu, S. High-sensitivity method for determination of tetrabromobisphenol-S and tetrabromobisphenol-A derivative flame retardants in great lakes herring gull eggs by liquid chromatographyatmospheric pressure photoionization-tandem mass spectrometry. Environ. Sci. Technol. 2010, 44 (22), 8615-21.

(9) Liu, L. Y.; Venier, M.; Salamova, A.; Hites, R. A. A Novel Flame Retardant in the Great Lakes Atmosphere: 3,3 ',5,5' 'Tetrabromobisphenol A Bis(2,3-dibromopropyl) Ether. Environ. Sci. Technol. Lett. 2016, 3 (5), 194-199. 
(10) Richardson, S. D.; Kimura, S. Y. Water Analysis: Emerging Contaminants and Current Issues. Anal. Chem. 2016, 88 (1), 546582.

(11) Qu, G.; Liu, A.; Wang, T.; Zhang, C.; Fu, J.; Yu, M.; Sun, J.; Zhu, N.; Li, Z.; Wei, G.; Du, Y.; Shi, J.; Liu, S.; Jiang, G. Identification of tetrabromobisphenol A allyl ether and tetrabromobisphenol A 2,3dibromopropyl ether in the ambient environment near a manufacturing site and in mollusks at a coastal region. Environ. Sci. Technol. 2013, 47 (9), 4760-4767.

(12) Liu, A. F.; Qu, G. B.; Yu, M.; Liu, Y. W.; Shi, J. B.; Jiang, G. B. Tetrabromobisphenol-A/S and Nine Novel Analogs in Biological Samples from the Chinese Bohai Sea: Implications for Trophic Transfer. Environ. Sci. Technol. 2016, 50 (8), 4203-4211.

(13) Li, F.; Jiang, B.; Nastold, P.; Kolvenbach, B. A.; Chen, J.; Wang, L.; Guo, H.; Corvini, P. F.-X.; Ji, R. Enhanced Transformation of Tetrabromobisphenol A by Nitrifiers in Nitrifying Activated Sludge. Environ. Sci. Technol. 2015, 49 (7), 4283-4292.

(14) Liu, A.; Qu, G.; Zhang, C.; Gao, Y.; Shi, J.; Du, Y.; Jiang, G. Identification of two novel brominated contaminants in water samples by ultra-high performance liquid chromatography-Orbitrap Fusion Tribrid mass spectrometer. J. Chromatogr. A 2015, 1377, 92-99.

(15) Liu, A.; Tian, Y.; Yin, N.; Yu, M.; Qu, G.; Shi, J.; Du, Y.; Jiang, G. Characterization of three tetrabromobisphenol-S derivatives in mollusks from chinese Bohai Sea: A strategy for novel brominated contaminants identification. Sci. Rep. 2015, 5.10.1038/srep11741

(16) Liu, J.; Wang, Y.; Jiang, B.; Wang, L.; Chen, J.; Guo, H.; Ji, R. Degradation, Metabolism, and Bound-Residue Formation and Release of Tetrabromobisphenol A in Soil during Sequential Anoxic-Oxic Incubation. Environ. Sci. Technol. 2013, 47 (15), 8348-8354.

(17) Bao, Y.; Niu, J. Photochemical transformation of tetrabromobisphenol A under simulated sunlight irradiation: Kinetics, mechanism and influencing factors. Chemosphere 2015, 134, 550-556.

(18) Li, F.; Wang, J.; Jiang, B.; Yang, X.; Nastold, P.; Kolvenbach, B.; Wang, L.; Ma, Y.; Corvini, P. F.-X.; Ji, R. Fate of Tetrabromobisphenol A (TBBPA) and Formation of Ester- and Ether-Linked Bound Residues in an Oxic Sandy Soil. Environ. Sci. Technol. 2015, 49 (21), 12758-12765.

(19) Wang, J.; Hou, Y.; Zhang, J.; Zhu, J.; Feng, Y. Transformation of 2,2',4,4'-tetrabromodiphenyl ether under UV irradiation: Potential sources of the secondary pollutants. J. Hazard. Mater. 2013, 263, 778783.

(20) Sun, F.; Kolvenbach, B. A.; Nastold, P.; Jiang, B.; Ji, R.; Corvini, P. F. Degradation and Metabolism of Tetrabromobisphenol A (TBBPA) in Submerged Soil and Soil-Plant Systems. Environ. Sci. Technol. 2014, 48 (24), 14291-9.

(21) Li, F.; Wang, J.; Nastold, P.; Jiang, B.; Sun, F.; Zenker, A.; Kolvenbach, B. A.; Ji, R.; Corvini, P. F.-X. Fate and metabolism of tetrabromobisphenol $\mathrm{A}$ in soil slurries without and with the amendment with the alkylphenol degrading bacterium Sphingomonas sp strain TTNP3. Environ. Pollut. 2014, 193, 181-188.

(22) An, T.; Zu, L.; Li, G.; Wan, S.; Mai, B.; Wong, P. K. One-step process for debromination and aerobic mineralization of tetrabromobisphenol-A by a novel Ochrobactrum sp $\mathrm{T}$ isolated from an e-waste recycling site. Bioresour. Technol. 2011, 102 (19), 9148-9154.

(23) Voordeckers, J. W.; Fennell, D. E.; Jones, K.; Haggblom, M. M. Anaerobic biotransformation of tetrabromobisphenol A, tetrachlorobisphenol A, and bisphenol A in estuarine sediments. Environ. Sci. Technol. 2002, 36 (4), 696-701.

(24) Ronen, Z.; Abeliovich, A. Anaerobic-aerobic process for microbial degradation of tetrabromobisphenol A. Appl. Environ. Microbiol. 2000, 66 (6), 2372-2377.

(25) Arbeli, Z.; Ronen, Z.; Diaz-Baez, M. C. Reductive dehalogenation of tetrabromobisphenol-A by sediment from a contaminated ephemeral streambed and an enrichment culture. Chemosphere 2006, 64 (9), 1472-1478.

(26) Shih, Y.-h.; Chou, H.-L.; Peng, Y.-H. Microbial degradation of 4monobrominated diphenyl ether with anaerobic sludge. J. Hazard. Mater. 2012, 213, 341-346.
(27) Chou, H.; Chang, Y.; Liao, Y.; Lin, C. Biodegradation of decabromodiphenyl ether (BDE-209) by bacterial mixed cultures in a soil/water system. Int. Biodeterior. Biodegrad. 2013, 85, 671-682.

(28) Ruppe, S.; Neumann, A.; Braekevelt, E.; Tomy, G. T.; Stern, G. A.; Maruya, K. A.; Vetter, W. Anaerobic transformation of compounds of technical toxaphene. 2. Fate of compounds lacking geminal chlorine atoms. Environ. Toxicol. Chem. 2004, 23 (3), 591-598.

(29) Ruppe, S.; Neumann, A.; Vetter, W. Anaerobic transformation of compounds of technical toxaphene. I. Regiospecific reaction of chlorobornanes with geminal chlorine atoms. Environ. Toxicol. Chem. 2003, 22 (11), 2614-2621.

(30) Kräutler, B.; Fieber, W.; Ostermann, S.; Fasching, M.; Ongania, K.-H.; Gruber, K.; Kratky, C.; Mikl, C.; Siebert, A.; Diekert, G. The Cofactor of Tetrachloroethene Reductive Dehalogenase of Dehalospirillum multivorans Is Norpseudo-B12, a New Type of a Natural Corrinoid. Helv. Chim. Acta 2003, 86 (11), 3698-3716.

(31) Peng, F. Q.; Ying, G. G.; Yang, B.; Liu, Y. S.; Lai, H. J.; Zhou, G. J.; Chen, J.; Zhao, J. L. Biotransformation of the fame retardant tetrabromobisphenol A (TBBPA) by freshwater microalgae. Environ. Toxicol. Chem. 2014, 33 (8), 1705-1711.

(32) Qu, R.; Feng, M.; Wang, X.; Huang, Q.; Lu, J.; Wang, L.; Wang, Z. Rapid Removal of Tetrabromobisphenol A by Ozonation in Water: Oxidation Products, Reaction Pathways and Toxicity Assessment. PLoS One 2015, 10, (10), e013958010.1371/journal.pone.0139580

(33) Xu, W.; Wang, X.; Cai, Z. Analytical chemistry of the persistent organic pollutants identified in the Stockholm Convention: A review. Anal. Chim. Acta 2013, 790, 1-13.

(34) Howard, P. H.; Muir, D. C. G. Identifying new persistent and bioaccumulative organics among chemicals in commerce. Environ. Sci. Technol. 2010, 44 (7), 2277-2285.

(35) Tian, Y.; Chen, J.; Ouyang, Y.; Qu, G.; Liu, A.; Wang, X.; Liu, C.; Shi, J.; Chen, H.; Jiang, G. Reactive extractive electrospray ionization tandem mass spectrometry for sensitive detection of tetrabromobisphenol A derivatives. Anal. Chim. Acta 2014, 814, 4954.

(36) Qu, G.; Liu, A.; Hu, L.; Liu, S.; Shi, J.; Jiang, G. Recent advances in the analysis of TBBPA/TBBPS, TBBPA/TBBPS derivatives and their transformation products. TrAC, Trends Anal. Chem. 2016, 83, $14-24$.

(37) Huang, D.; Zhao, H.; Liu, C.; Sun, C. Characteristics, sources, and transport of tetrabromobisphenol $\mathrm{A}$ and bisphenol $\mathrm{A}$ in soils from a typical e-waste recycling area in South China. Environ. Sci. Pollut. Res. 2014, 21 (9), 5818-5826.

(38) Ruppe, S.; Neumann, A.; Diekert, G.; Vetter, W. Abiotic transformation of toxaphene by superreduced vitamin B-12 and dicyanocobinamide. Environ. Sci. Technol. 2004, 38 (11), 3063-3067.

(39) Gaul, S.; Von der Recke, R.; Tomy, G.; Vetter, W. Anaerobic transformation of a technical brominated diphenyl ether mixture by super-reduced vitamin B-12 and dicyanocobinamide. Environ. Toxicol. Chem. 2006, 25 (5), 1283-1290.

(40) von der Recke, R; Vetter, W. Synthesis and characterization of 2,3-dibromopropyl-2,4,6-tribromophenyl ether (DPTE) and structurally related compounds evidenced in seal blubber and brain. Environ. Sci. Technol. 2007, 41 (5), 1590-1595.

(41) Ballesteros-Gómez, A.; de Boer, J.; Leonards, P. E. A novel brominated triazine-based flame retardant (TTBP-TAZ) in plastic consumer products and indoor dust. Environ. Sci. Technol. 2014, 48 (8), 4468-4474.

(42) Vanhaecke, L.; Van Meulebroek, L.; De Clercq, N.; Bussche, J. V. High resolution orbitrap mass spectrometry in comparison with tandem mass spectrometry for confirmation of anabolic steroids in meat. Anal. Chim. Acta 2013, 767, 118-127.

(43) Arbeli, Z.; Ronen, Z. Enrichment of a microbial culture capable of reductive debromination of the flame retardant tetrabromobisphenol-A, and identification of the intermediate metabolites produced in the process. Biodegradation 2003, 14 (6), 385-395.

(44) Zhang, C.; Li, Z.; Suzuki, D.; Ye, L.; Yoshida, N.; Katayama, A. A humin-dependent Dehalobacter species is involved in reductive 
debromination of tetrabromobisphenol A. Chemosphere 2013, 92 (10), 1343-8.

(45) Wang, X.; Hu, X.; Zhang, H.; Chang, F.; Luo, Y. Photolysis Kinetics, Mechanisms, and Pathways of Tetrabromobisphenol A in Water under Simulated Solar Light Irradiation. Environ. Sci. Technol. 2015, 49 (11), 6683-90.

(46) Nakao, T.; Akiyama, E.; Kakutani, H.; Mizuno, A.; Aozasa, O.; Akai, Y.; Ohta, S. Levels of Tetrabromobisphenol A, Tribromobisphenol A, Dibromobisphenol A, Monobromobisphenol A, and Bisphenol A in Japanese Breast Milk. Chem. Res. Toxicol. 2015, 28 (4), 722-728.

(47) Akiyama, E.; Kakutani, H.; Nakao, T.; Motomura, Y.; Takano, Y.; Sorakubo, R.; Mizuno, A.; Aozasa, O.; Tachibana, K.; Doi, T.; Ohta, S. Facilitation of adipocyte differentiation of 3T3-L1 cells by debrominated tetrabromobisphenol A compounds detected in Japanese breast milk. Environ. Res. 2015, 140, 157-164.

(48) Feng, Y.; Colosi, L. M.; Gao, S.; Huang, Q.; Mao, L. Transformation and Removal of Tetrabromobisphenol A from Water in the Presence of Natural Organic Matter via LaccaseCatalyzed Reactions: Reaction Rates, Products, and Pathways. Environ. Sci. Technol. 2013, 47 (2), 1001-1008.

(49) Ruan, T.; Wang, Y.; Wang, C.; Wang, P.; Fu, J.; Yin, Y.; Qu, G.; Wang, T.; Jiang, G. Identification and Evaluation of a Novel Heterocyclic Brominated Flame Retardant Tris(2,3-dibromopropyl) Isocyanurate in Environmental Matrices near a Manufacturing Plant in Southern China. Environ. Sci. Technol. 2009, 43 (9), 3080-3086.

(50) Glod, G.; Angst, W.; Holliger, C.; Schwarzenbach, R. P. Corrinoid-mediated reduction of tetrachloroethene, trichloroethene, and trichlorofluoroethene in homogeneous aqueous solution: Reaction kinetics and reaction mechanisms. Environ. Sci. Technol. 1997, 31 (1), 253-260.

(51) Covaci, A.; Voorspoels, S.; Abdallah, M. A.-E.; Geens, T.; Harrad, S.; Law, R. J. Analytical and environmental aspects of the flame retardant tetrabromobisphenol-A and its derivatives. $J$. Chromatogr. A 2009, 1216 (3), 346-363.

(52) Zhou, J.; Chen, J.; Liang, C.; Xie, Q.; Wang, Y.; Zhang, S.; Qiao, X.; Li, X. Quantum Chemical Investigation on the Mechanism and Kinetics of PBDE Photooxidation by center dot OH: A Case Study for BDE-15. Environ. Sci. Technol. 2011, 45 (11), 4839-4845.

(53) Cheng, J.; Mao, L.; Zhao, Z.; Shen, M.; Zhang, S.; Huang, Q.; Gao, S. Bioaccumulation, depuration and biotransformation of 4,4 '-dibromodiphenyl ether in crucian carp (Carassius auratus). Chemosphere 2012, 86 (5), 446-453.

(54) Boxtel, A. L. v.; Kamstra, J. H.; Cenijn, P. H.; Pieterse, B.; Wagner, M. J.; Antink, M.; Krab, K.; Burg, B. v. d.; Marsh, G.; Brouwer, A.; Legler, J. Microarray analysis reveals a mechanism of phenolic polybrominated diphenylether toxicity in zebrafish. Environ. Sci. Technol. 2008, 42 (5), 1773-1779.

(55) Dingemans, M. M.; de Groot, A.; van Kleef, R. G.; Bergman, A.; van den Berg, M.; Vijverberg, H. P.; Westerink, R. H. Hydroxylation increases the neurotoxic potential of BDE-47 to affect exocytosis and calcium homeostasis in PC12 cells. Environ. Health Perspect. 2008, 116 (5), 637-43.

(56) Wang, X. B.; Wang, Y.; Chen, J. W.; Ma, Y. Q.; Zhou, J.; Fu, Z. Q. Computational Toxicological Investigation on the Mechanism and Pathways of Xenobiotics Metabolized by Cytochrome P450: A Case of BDE-47. Environ. Sci. Technol. 2012, 46 (9), 5126-5133.

(57) Debenest, T.; Gagne, F.; Petit, A. N.; Andre, C.; Kohli, M.; Blaise, C. Ecotoxicity of a brominated flame retardant (tetrabromobisphenol A) and its derivatives to aquatic organisms. Comp. Biochem. Physiol., Part C: Toxicol. Pharmacol. 2010, 152 (4), 407-412. 